\title{
BESPRECHUNGSAUFSATZ
}

\section{Menschenrechte, Klimaschutz und der ökonomisch begründete Begriff der Freiheit ${ }^{1}$}

\author{
Von Hermann Weber
}

\section{Die Bedrohung und Erosion des individuellen Grundstatus im Ausgang des Jahrhunderts}

Einige fundamentale Fragen des Klimaschutzes, die auf den Konferenzen der Vereinten Nationen in Rio de Janeiro (1992), in Berlin (1995) und zuletzt in Kyoto (1997) aufgeworfen wurden, zwingen dazu, Grundsatzfragen des Umweltschutzes in veränderter Perspektive zu sehen, ihn insbesondere als Teil der Menschenrechte anzuerkennen und seine Probleme in den Zusammenhang mit dem allenthalben beobachteten und erfahrenen Wandel der Arbeitswelt und der Lebensbedingungen der Menschen zu stellen. Der Verlauf der Umwelt- und Klimaschutzkonferenzen der Vereinten Nationen und ihr hinter den Herausforderungen der Zeit weit zurückbleibendes Ergebnis zwingen dazu, nach den tieferen Ursachen zu fragen, was die Staaten und Regierungen daran hindert, dem gesellschaftlichen und technologischen Wandel mit angemessenen und menschengerechten Lösungen zu begegnen. Die gestellte Frage soll von einem Phänomen aus betrachtet und untersucht werden, das im Ausgang dieses Jahrhunderts ständig an Brisanz zugenommen hat, ohne zugleich jenes Gewicht in der realen Politik erlangt zu haben, das ihm angesichts seiner Bedeutung und Größenordnung zukommt: der Bedrohung der individuellen Freiheit als einem zentralen Begriff der Menschenrechte durch zunehmend irreparable Beschädigungen der elementaren Lebensbedingungen in der natürlichen Umwelt.

Die Forderung nach verstärktem Schutz der Menschenrechte gehört heute zum unverzichtbaren Bestand jeder demokratisch legitimierten Politik. Nichtdemokratisch legitimierte Regime stehen unter dem Druck, sich über die Einhaltung menschenrechtlicher Mindest-

zugleich eine Besprechung der "Dokumente zur Geschichte der Vereinigten Staaten", herausgegeben von Herbert Schambeck, Helmut Widder und Marcus Bergmann, Verlag Duncker \& Humblot, Berlin 1993, XXVII, 729 S., geb., DM 98,-- 
standards zu behaupten, weil sie andernfalls als Partner in den internationalen Beziehungen nicht voll anerkannt werden. Solche Regime sind gehalten, in jedem Einzelfall der Abweichung vom internationalen Menschenrechtsstandard eine Begründung zu liefern. Diesen Standard bestimmen heute die internationalen Menschenrechtskonventionen von 1966, darüber hinaus eine Reihe von Spezialübereinkommen, etwa zur Verhütung von Folter (1984), Geiselnahme (1979), Terrorismus (1977) und Rassismus (1966), die Flüchtlingskonventionen von 1951 und 1967 sowie die vier Genfer Rotkreuzkonventionen von 1949 zum Schutz der Verwundeten und Kranken im Land- und Seekrieg, der Kriegsgefangenen sowie der Zivilpersonen in bewaffneten Konflikten. Zwei 1977 abgeschlossene Zusatzprotokolle zu den Genfer Konventionen passen den Mindeststandard an die Gegebenheiten der Kriegführung in internationalen und nichtinternationalen bewaffneten Konflikten an. Durch diese und weitere internationale Übereinkommen soll sichergestellt werden, daß für jedermann überall und zu jeder Zeit eine mit seiner Menschenwürde vereinbare Existenz unabhängig von seiner Herkunft, seinem Glauben, seiner politischen Überzeugung, seiner Hautfarbe und seinem Geschlecht nach Grundsätzen der Gleichbehandlung und der Rechtsstaatlichkeit gewährleistet ist und zusätzlich in Kriegszeiten ein Mindeststandard, der unter keinen politischen, militärischen oder anderen "kriegsbedingten" Gesichtspunkten unterschritten werden darf. Die Europäische Union hat die Aufnahme der Slowakei und der Türkei in die Kandidatenliste für eine Mitgliedschaft in der EU gerade mit dem Argument abgelehnt, die Politik dieser Staaten gegenüber Andersdenkenden und nationalen Minderheiten entspreche nicht dem international gültigen Mindeststandard.

Trotz eindeutiger Regeln erscheint indessen nicht nur die Menschenrechtspolitik der Slowakei und der Türkei widersprüchlich. Die Beharrlichkeit, mit der sich die ehemaligen Kriegsparteien in Bosnien gegen die Erfüllung der im Friedensabkommen von Dayton/USA 1995 übernommenen Verpflichtungen sträuben (Boykott der Rückkehr der Flüchtlinge in ihre Heimatorte, Diskriminierung ethnischer Minderheiten, Wahlboykott, Weigerung der politischen Repräsentanten, mit Vertretern anderer Volksgruppen zusammenzuarbeiten und anderes mehr), beweist, in welchem Maße die Frage der Durchsetzung eines effizienten Menschenrechtsschutzes in der Praxis auf Widerstände stößt.

Auch die Bundesrepublik Deutschland muß sich immer wieder der Tatsache stellen, daß der internationale Menschenrechtsstandard in praxi ein Lippenbekenntnis ist. Eine Resolution des Deutschen Bundestages zur Tibet-Frage vom Juni 1996, in der das Vorgehen der chinesischen Regierung gegen die tibetische Freiheitsbewegung als Verstoß gegen die Menschenrechte gerügt worden war, hat die deutsch-chinesischen Beziehungen zeitweise schwer belastet. Ein Urteil des Berliner Landgerichts vom April 1997, das die Regierung in Teheran der Beteiligung an einem politischen Mord überführt hatte, hat die Bundesregierung gezwungen, den "kritischen Dialog" mit der iranischen Regierung abzubrechen. Die Bundesregierung hatte vergeblich gehofft, den Iran in der Frage der Verfolgung politischer Dissidenten langfristig zu einer Kehrtwendung und in der Frage der gegen den Schriftstel- 
ler Salman Rushdie ausgesprochenen Morddrohung zu einem menschengerechten Procedere bewegen zu können.

Die den Staaten prinzipiell abverlangte Einbeziehung des menschenrechtlichen Mindeststandards in das gesamte internationale Beziehungsgeflecht könnte deshalb am Ausgang des Jahrhunderts als die entscheidende zivilisatorische Errungenschaft gelten, wenn sie nicht in der Praxis durch grobe und gröbste Menschenrechtsverletzungen konterkariert würde. Die menschenrechtlichen Mindestnormen sind noch in zu vielen Fällen Gegenstand des internationalen Streites, als daß sie bereits als eine sichere Wegmarke für das Staatenverhalten im 21. Jahrhundert erkannt werden können. Die Passivität und Indifferenz, mit der die Öffentlichkeit auf Rassenkonflikte und weltanschauliche Fundamentalismus-Konflikte in Afrika, Afghanistan, Indien, Nordirland, im Kaukasus und auf dem Balkan reagiert - Konflikte, in denen die Zivilbevölkerung in zunehmendem Maße mit den Mitteln des Terrors und des Gegenterrors zur Geisel von Fanatikern gemacht wird -, lassen den Menschenrechtsschutz zu einer bloßen Rhetorik verkommen. Der Weg in eine zunehmende Radikalisierung der Formen und Methoden, in denen politische Auseinandersetzungen im Innern der Staaten ausgetragen werden, ist seit längerer Zeit zu erkennen. In Äthiopien und in Eritrea hat die Staatengemeinschaft einem grausamen Bürgerkrieg über Jahrzehnte in vollständiger Untätigkeit zugesehen. In Moçambique wurde das Weltgewissen noch Jahre nach Beendigung der Kampfhandlungen zwischen Regierung und Rebellen nicht aufgerüttelt, als die körperliche Verstümmelung der Zivilbevölkerung durch Landminen weiterging und ein sofortiges Einschreiten der Staatengemeinschaft unter den Kategorien des Menschenschutzes gefordert hätte. In Osttimor duldet die UNO die Terrorherrschaft Indonesiens in aller Offenheit, während die USA mit Djakarta über die Ausbeutung der Rohstoffe Osttimors vertragliche Vereinbarungen treffen. Die zur Dauereinrichtung in Algerien gewordenen Massaker an der Zivilbevölkerung werden durch kein internationales Einschreiten beendet, weil Ölinteressen und die Furcht vor einer Regierungsübernahme durch die "Islamische Heilsfront" ein gemeinsames Vorgehen der Staatengemeinschaft inopportun erscheinen lassen.

$\mathrm{Zu}$ der Bedrohung und der Erosion von Leben und Freiheit des einzelnen durch direkte Gewalt gesellen sich die Freiheitsgefährdungen durch wirtschaftliche und gesellschaftliche Veränderungen, die der technologische Wandel und der grenzenlose Wettbewerb, den er ermöglicht, hervorgerufen hat. Dieser Bedrohung sind die traditionellen Verhaltens-, Planungs- und Steuerungskonzepte nicht mehr gewachsen. Viele funktionierende Strukturen sind bereits zerstört, ohne daß das Neue, das erst in Ansätzen sichtbar wird, die Menschen mit Hoffnungen erfüllt. Mit der Zerstörung traditioneller Arbeits- und Lebensformen geht ein Autoritätsverfall einher, der in ganz neuen Gewaltszenarien und Kriminalitätsformen sich Bahn bricht. Verbindet sich dieser Autoritätsverfall mit traditionellen Defiziten, wie Armut, Analphabetentum, Kinderarbeit, Prostitution, Korruption und Menschenhandel, so 
werden der Freiheitsraum des einzelnen und sein menschenrechtlicher Grundstatus noch einmal zusätzlich geschwächt und ausgehöhlt.

So gesehen, sind Bedrohung und Erosion des individuellen Grundstatus, auf den jedermann nach universell gültigen Rechtsprinzipien Anspruch hat, die erweiterte Dimension einer schleichenden Veränderung aller gesellschaftlichen Rahmenbedingungen, für die die belastete Umwelt und die Bedrohung des Weltklimas nur der stärkste Ausdruck sind. Allerdings läßt sich die Bedrohung des Individuums durch sichtbare und meßbare Veränderungen der natürlichen Umweltkonstanten mit wirtschaftlichen und gesellschaftlichen Anpassungsprozessen allein nicht erklären. Phänomene wie Überalterung, Urbanisation und Landflucht, Migration und durch Krieg und Bürgerkrieg ausgelöste Flüchtlingsbewegungen haben eine lange Tradition. Erst das Ausmaß, das sie in der Gegenwart angenommen haben, läßt sie auch als Umweltprobleme erscheinen, denen die Staaten mit traditionellen Kategorien und Instrumenten der Steuerung und Gesetzgebung nur unzureichend beikommen. Erst vor diesem Hintergrund erhalten die Ergebnisse - besser: Nichtergebnisse - der Umwelt- und Klimakonferenzen der Vereinten Nationen ihr spezifisches Bedrohungsprofil für den menschenrechtlichen Grundstatus des einzelnen.

\section{Die bedrohte Umwelt als neue Dimension der Menschenrechte}

Auf den Umwelt- und Klimakonferenzen der Vereinten Nationen sind die von einzelnen Wissenschaftlern bereits für unumkehrbar erklärten negativen Auswirkungen jeder weiteren uneingeschränkten Nutzung der fossilen Rohstoffe auf das Klima der Erde drastisch beschrieben worden. Trotz allgemeiner Zustimmung zur Zustandsbeschreibung stoßen die daraus gezogenen Folgerungen jedoch auf zahllose Wenn und Aber. Selbst die naheliegenden Konsequenzen werden als Handlungsbedarf nicht anerkannt und für die Politik nicht nutzbar gemacht. Staaten, die zu den Hauptverursachern des Treibhauseffektes zählen, weigern sich, einschneidende Korrekturen des politischen Kurses vorzunehmen. Die an Zahl und Intensität sich häufenden Umweltkatastrophen werden im besten Fall als Kostenfaktoren in die wirtschaftliche Betriebsbilanz der betroffenen Staaten eingestellt und provozieren kein Umdenken der Politik. Das "big business" reagiert mit harter Ablehnung und Widerstand auf alle Gegenstrategien, wie sie die UNO z.B. mit dem Konzept der "nachhaltigen Entwicklung" (sustainable development) unterstützt. Die Protagonisten der Besitzstandswahrung werden nicht müde, solche Konzepte als utopisch und unverhältnismäßig zu marginalisieren, um ihre Verwirklichung schon im Ansatz unmöglich zu machen.

Cum grano salis gilt diese Situationsbeschreibung für alle Staaten, für die großen Industriestaaten ebenso wie für die Schwellenländer und die ärmsten Staaten Afrikas und Lateinamerikas. In den sich gerade erst industrialisierenden Schwellenländern aber hat der Verzicht auf jede Technologiefolgenabschätzung und Umweltverträglichkeitsprüfung im Zuge 
der totalen wirtschaftlichen Umgestaltung die gravierendsten Auswirkungen. Dort wurde der forcierte industrielle Fortschritt mit den größten Umweltschäden erkauft mit der Folge, daß mehrere südostasiatische Staaten, wie Malaysia und Indonesien, heute am Rande einer Katastrophe stehen. Es sind zugleich diejenigen Staaten, die auch in der Frage der Sicherung der Menschenrechte im klassischen Sinne das Schlußlicht der Staaten bilden. Dies gilt auch für China. Einer Studie der Vereinten Nationen zufolge liegen 13 von 15 der am stärksten durch Luftverschmutzung belasteten Großstädte in Asien.

In den hochindustrialisierten Staaten des Nordens, in Europa und in den Vereinigten Staaten, stellt sich die Frage der Gefährdung von Leben und Freiheit des einzelnen in einer anderen Perspektive. Dort steht der Mensch seit mehreren Jahren unter dem Druck eines sich ständig verschärfenden internationalen Wettbewerbs von Arbeit, Produktion und Dienstleistungen. Noch halten sich die negativen Auswirkungen auf den traditionellen Menschenrechtsstandard in Grenzen, doch hat der technische und wirtschaftliche Strukturwandel auch in den hochindustrialisierten Staaten Folgen gezeitigt, wie man sie bisher in prosperierenden Ökonomien nicht kannte: Dauerarbeitslosigkeit in zweistelligen Prozentzahlen, Anstieg der Drogen- und Alkoholabhängigkeit, zunehmende rassistische Einstellungen sowie Angriffe und Kriminalität in neuen Formen und Größenordnungen haben ein Klima hinterlassen, das für einen effektiven Menschenrechtsschutz nicht günstig ist. Kommen dazu diskriminierende und repressive Praktiken des Staates gegenüber Personen, deren Status labil ist, weil er durch periodische Aufenthaltsüberprüfungen und Arbeitsverbote in Frage gestellt wird, so registrieren auch nicht direkt davon Betroffene jedes politische Handlungsdefizit als latenten Angriff auf ihr Menschenrecht.

In den Industriestaaten des Nordens war das hohe Niveau der sozialen Sicherungen und des individuellen Grundstatus das Spiegelbild ihrer erfolgreichen politischen und wirtschaftlichen Entwicklung nach Beendigung des Zweiten Weltkrieges. Der Koreakrieg und der Vietnamkrieg haben dieser Entwicklung noch einmal zusätzliche Schubkraft gegeben. Die überproportionale Ressourcennutzung ist Teil dieser Erfolgsgeschichte. Dieser Raubbau an den nicht regenerativen Ressourcen der Erde kann indes nicht in dem Maße fortgesetzt werden, wie er bisher betrieben wurde, ohne daß das gesamte ökologische Gleichgewicht schweren Schaden nimmt. Den Raubbau verbietet schon der Ressourcenbedarf der nichtindustrialisierten Länder unter den Kategorien der Gleichbehandlung in der Zukunft. Für die Entwicklungsländer ist ein Wasserbedarf von fünf Litern pro Person und Tag für die Zukunft errechnet worden. Im Vergleich dazu wird der Wasserverbrauch in Europa mit einem Multiplikator 50 und in den USA mit 100 angegeben. Allein der $\mathrm{CO}_{2}$-Ausstoß der USA ist mit einem Anteil von 22,4\% an den globalen Kohlendioxyd-Emissionen größer als die entsprechenden Anteile Chinas (13,4\%) und Rußlands (7,1\%) zusammengenommen, obgleich die USA nur einen Anteil von 1/6 der Bevölkerung dieser Staaten stellen. 
Im Angesicht der Defizite der individuellen Statussicherung in umweltpolitischer Perspektive und der negativen Umweltprognosen erscheint der Widerstand der Vereinigten Staaten auf den Umwelt- und Klimakonferenzen der Vereinten Nationen, eine umweltpolitische Kehrtwende einzuleiten, anachronistisch. Die USA, die selbst die bescheidenen Vorschläge der Europäischen Staaten zur Begrenzung des $\mathrm{CO}^{2}$-Ausstoßes nicht akzeptiert haben, folgen den Argumenten ihrer Großunternehmen, die in jeder Beschränkung der Energienutzung aus Gründen des Klimaschutzes einen unzulässigen Eingriff des Staates in die Gesetze des freien Marktes erblicken. Bemerkenswert ist dabei, daß die breite Öffentlichkeit in den Vereinigten Staaten nach einer jüngst unternommenen Umf rage diese Argumentation nicht teilt. $65 \%$ der befragten Bürger sprachen sich danach für eine Reduzierung der Kohlendioxyd-Emissionen auf nationaler Grundlage aus, ungeachtet dessen, wie andere Staaten auf diese Herausforderung reagieren.

Ein langsamer Bewußtseinswandel ist deshalb auch in den USA nicht auszuschließen, es bleibt aber abzuwarten, ob sich dieser Wandel in Zukunft fortsetzen wird. Der generelle Verweis auf Macht, Gruppenegoismus und Interessenantagonismus erklärt nur zum Teil die ablehnende Haltung der Vereinigten Staaten auf den Umwelt- und Klimakonferenzen der Vereinten Nationen. Er erklärt vor allem nicht die Härte, mit der sich die USA allen Kurskorrekturen in der Klimapolitik in den Weg stellen. Die USA sind nach der politischen Wende in Ost- und Mitteleuropa 1989/90 die einzig verbliebene Großmacht, deren ökonomisches System für alle diejenigen Staaten zum Vorbild geworden ist, die aus dem Zusammenbruch des Ostblocks hervorgegangen sind. Als junge und erstmals unabhängige Völkerrechtssubjekte sehen sie in der Erfolgsgeschichte der USA das Muster, nach dem sie ihre eigene wirtschaftliche und politische Entwicklung gestalten und befördern zu können glauben. Als erstmals in das globale Wirtschaftssystem eingebundene Staaten haben die Newcomer auch wenig Möglichkeiten, die politischen und wirtschaftlichen Weichen anders als von diesem System vorgegeben zu stellen. Auf der anderen Seite erlaubt es die Vorbildfunktion den USA, zusätzlich zu ihrem politischen, militärischen und wirtschaftlichen Gewicht Vorstellungen durchzusetzen, mit denen sie heute viele Staaten auf ihrer Seite wissen.

\section{Das Credo der freien Eigentumsverfügung als Bedingung des individuellen Freiheitsanspruchs?}

Die Freiheit der Wirtschaft und des Welthandels und freies Unternehmertum rangieren an oberster Stelle der politischen Prioritäten, denen sich heute die Staatengemeinschaft, angeführt von den Vereinigten Staaten, verpflichtet fühlt. Demgegenüber haben die Menschenrechte und der Umweltschutz keinen vergleichbar hohen Stellenwert, auch wenn die öffentliche Diskussion darüber seit der ersten Umweltkonferenz der Vereinten Nationen in Stockholm 1972 stetig zugenommen hat. Mit der ökonomischen Vorbildfunktion der USA 
stellt sich die Frage, ob die Vereinigten Staaten langfristig auch die Leitlinien für den internationalen Umwelt- und Klimaschutz für alle Staaten mehr oder weniger verbindlich vorgeben werden. Für ein anderes internationales Leitprinzip, die Demokratie, ist die Frage problemlos zu beantworten: Die Demokratie, die keine amerikanische Erfindung ist, obgleich die USA zu ihrer Verbreitung einen entscheidenden Beitrag geleistet haben, hat viele Wurzeln. Sie ist heute als allgemeingültiges politisches Gestaltungsprinzip universal anerkannt, wenn auch nicht überall gleich überzeugend praktiziert. Das globale, zunehmend nach rein marktwirtschaftlichen Gesetzen operierende Wirtschaftssystem ist jedoch amerikanischen Ursprungs und verlangt nach anderen Antworten. Es ist in der Erfolgsgeschichte der Vereinigten Staaten begründet und kann von dieser nicht getrennt werden, wenn es in seiner Funktion verstanden werden soll.

Die amerikanische Erfolgsgeschichte weist auf einen zentralenWiderspruch der amerikanischen Haltung auf den Umwelt- und Klimakonferenzen der Vereinten Nationen hin: auf die Tatsache, daß die USA seit jeher für sich in Anspruch nehmen, Verteidiger der Menschenrechte und geistig- moralischer Träger des Freiheitsgedankens zu sein, ihren Widerstand gegen notwendige Korrekturen in der Umwelt- und Klimapolitik, einem zentralen Anliegen der individuellen Lebens- und Freiheitssicherung, jedoch mit einem Prinzip verteidigen, das dieses Lebens- und Freiheitsrecht des einzelnen gerade bedroht: mit dem Anspruch auf uneingeschränkte Entfaltung individueller wirtschaftlicher Unternehmensfreiheit. Das Paradoxon der amerikanischen Position erklärt sich aus der Verfassungsgeschichte der Vereinigten Staaten, in der die Frage des Individualschutzes wiederholt die inneren Auseinandersetzungen bestimmt hatte, zuletzt aber und mit unterschiedlicher Begründung immer der ökonomischen Interessenlage untergeordnet wurde. Von dieser Interessenlage hat der amerikanische Freiheitsanspruch seine spezifische Prägung erhalten.

Über die Grundlagen des amerikanischen Verfassungsdenkens informiert eine Publikation, die Herbert Schambeck, Helmut Widder und Marcus Bergmann 1993 unter dem Titel "Dokumente zur Geschichte der Vereinigten Staaten" herausgegeben haben. Diese Sammlung stellt alle wichtigen Dokumente vor, die im Verlauf der amerikanischen politischen Entwicklung die Eckwerte des amerikanischen Verfassungsdenkens bestimmt haben: außer eigentlichen Verfassungsdokumenten auch Verfassungsentwürfe, Verträge, Vertragsentwürfe, Tagebucheinträge maßgeblicher Entscheidungsträger, Gerichtsentscheidungen, Reden und Vorträge politischer Repräsentaten und privater Meinungsführer u.a.m. Für den amerikanischen Verfassungsbegriff, das Freiheits-Credo, und die politisch-wirtschaftlichen Grundüberzeugungen, wie sie sich an zentralen Weichenstellungen der politischen Auseinandersetzung herausgebildet haben, sind die frühen Dokumente besonders aufschlußreich. Aus ihnen erschließt sich dem Leser der ideelle Grundgedanke des amerikanischen politischen Kategoriensystems und wie er mit den Prinzipien des internationalen Menschenrechtsschutzes, nicht zuletzt in seiner umweltpolitischen Dimension, korreliert. 
Die Vereinigten Staaten haben in internationale Konflikte, in denen sie ihre eigenen Interessen tangiert sahen, häufig in einer Weise eingegriffen, die ihre Intervention eher als Kreuzzug denn als eine von der Völkerrechtsordnung gebilligte Sanktion erscheinen ließ. Dies gilt nicht nur für die Interventionen des 19. und 20. Jahrhunderts in Zentralamerika und in der Karibik bis zur Gründung der Organisation der Vereinten Nationen, sondern auch für den Zeitraum danach, als die Primärzuständigkeit für alle Formen direkter Gewaltanwendung zwischen den Staaten auf den Sicherheitsrat übergegangen war. Diese UNZuständigkeit haben die USA immer umgangen und ihren eigenen politischen Vorstellungen angepaßt, wenn sie den Eindruck hatten, die amerikanischen Grundüberzeugungen würden nicht hinreichend in der Zuständigkeit des Sicherheitsrates erfaßt oder in dessen Entscheidungen berücksichtigt. Dies gilt z.B. für die Auseinandersetzungen mit Cuba bis zum heutigen Tage wegen der von Fidel Castro 1960 dekretierten Enteignung der amerikanischen Zuckerplantagen. Es gilt dies für den Vietnam-Konflikt mit Hanoi wegen der von den USA verweigerten Wiedervereinigung mit dem damaligen (nichtkommunistischen) Südvietnam - ein Konflikt, den die Vereinigten Staaten niemals vor den Sicherheitsrat gebracht haben -, und es gilt dies auch für den Golfkonflikt 1990/91, in dem es nur vordergründig um die Verteidigung Kuwaits gegen den Agressor Irak gegangen ist, in Wahrheit um den Schutz der amerikanischen Ölinteressen in Saudiarabien und in den Anrainerstaaten auf der arabischen Halbinsel. Diese Interventionen hatten alle eines gemeinsam: Sie wurden von den USA als Angriff auf ihre politisch-wirtschaftlichen Grundpositionen verstanden, unter ihnen das Prinzip, daß die freie Verfügung über privates Eigentum die entscheidende Bedingung für die Ausübung der Freiheitsrechte des einzelnen ist.

Aus dem Credo der freien Eigentumsverfügung leiten die USA das Dogma von der prinzipiell uneingeschränkten Güter- und Kapitalverkehrsfreiheit ab - eine Forderung, der sie soziale und kulturelle Kategorien in Staat und Gesellschaft unterordnen. Dem entspricht es, daß der soziale Schutz des einzelnen, wie er in Europa in unterschiedlichen Formen zur Korrektur eines ungebremsten Betätigungsdranges des freien Unternehmertums seit langem eingeführt ist, in denUSA, von der kurzen Periode des Roosevelt'schen "New Deal" in den dreißiger Jahren abgesehen, keine Nachahmung gefunden hat. Auch sozialpolitische Programme, wie das von Lyndon B. Johnson initiierte "Anti-Poverty-Program" erreichten niemals Rang und Ausmaß der europäischen Sozialgesetzgebung. Bis heute sind die USA auch dem UN-Menschenrechtspakt für soziale, wirtschaftliche und kulturelle Rechte nicht beigetreten.

Das amerikanische Menschenrechtsverständnis, das über den Schutz klassischer Bürgerfreiheiten nicht hinausreicht, bleibt deutlich hinter dem europäischen Standard zurück, der in den sozialen Grundrechten, durch eine umfangreiche Gesetzgebung materiell- und verfahrensrechtlich abgesichert, einen Bestandteil des modernen Menschenrechtsschutzes sieht. Zum erweiterten Sozialstandard in Europa gehört auch eine Umweltschutzgesetzgebung, die nicht in allen Mitgliedstaaten der EU gleich weit entwickelt ist, der jedoch der 
Gedanke gemeinsam ist, daß Leben und Freiheit des einzelnen heute nur gesichert erscheinen, wenn Industrieansiedlung umweltverträglich erfolgt und alle vorhandenen umweltschädlichen Einrichtungen und Produktionsmethoden den Erfordernissen eines wirksamen Umwelt- und Klimaschutzes angepaßt werden. Dabei ist die EU besonders bemüht, die Kategorien der Umweltverträglichkeit, die Schadensintensität, die Langzeitwirkungen von Umwelteinflüssen und die Möglichkeiten, problematische Einwirkungen auf das ökologische Gleichgewicht durch unproblematische zu ersetzen, dem wissenschaftlichen Forschungs- und Erkenntnisstand anzupassen.

Die Herausgeber betonen zu Recht, daß auch in den USA Staat und Politik nicht Selbstzweck sind. Beide Begriffe werden auf den Menschen bezogen, und die Anpassung der Verfassung an eine veränderte Wirklichkeit ist immer auch als Beitrag zur Statusverbesserung des einzelnen verstanden worden. Trotzdem ist es in den USA auch unter den engen Kategorien klassischer Bürgerrechte zu schweren Verfassungsbrüchen gekommen. Die Internierung Tausender amerikanischer Bürger japanischer Herkunft :ohne gesetzliche Grundlage während des Zweiten Weltkrieges und die Verfolgung und Ächtung amerikanischer Wissenschaftler, Schriftsteller, Schauspieler und Filmschaffender durch den Kongreßausschuß "gegen unamerikanische Umtriebe" in den Jahren des Kalten Krieges unter Mißachtung der in der Verfassung garantierten Grundrechte gehören noch heute zu den traumatischen Erfahrungen der davon betroffenen Menschen und ihrer Angehörigen.

\section{4. Ökonomisch begründetes Freiheitsrecht gegen Menschenrecht}

Fragt man nach den Ursachen für die in den USA verbreitete latent ablehnende Haltung, die Menschenrechte als einen weiten Rechtskanon zu begreifen, in dem alle Regeln enthalten sind, die den Kern des jedermann zustehenden Lebens- und Freiheitsraumes zu sichern geeignet sind, so zeigen die frühen Verfassungsdokumente, daß schon der Schutz der engeren "klassischen" Menschenrechte in den sich neu bildenden politischen Gemeinwesen Amerikas nicht übereinstimmend anerkannt war. Freiheitsrechte wurden von den eingewanderten Siedlern als spezifische Gruppenrechte zum eigenen Nutzen betrachtet und von ihnen unter diesem Vorzeichen zur Anwendung gebracht. Freiheitsrechte wurden als unentziehbare Rechte des freigeborenen Menschen beliebiger Herkunft und beliebigen Glaubens definiert. Als Zugangsrechte für wirtschaftliche Vorteile waren sie aber nur eingewanderten weißen Siedlern und ihren Nachkommen vorbehalten. Schwarze Sklaven und Angehörige der indianischen Völker waren von allen Freiheitsrechten ausgeschlossen. Dies war deshalb bemerkenswert, weil die ersten Siedlergemeinschaften das Selbstverständnis verband, in bewußter Abkehr von gesellschaftlicher Diskriminierung und religiöser und politischer Verfolgung Europa verlassen und in Amerika "God's own country" gesucht zu haben. 
Die Dokumente zur amerikanischen Verfassungsgeschichte zeigen überzeugend, in welchem Grade die Verengung einer weltumspannenden ethischen Idee auf eine weitgehend auf Siedlerinteressen zugeschnittene wirtschaftliche Rechtfertigungsideologie schon die ersten Auseinandersetzungen der amerikanischen Neubürger mit der britischen Krone ausgezeichnet hat. Lange vor der Bostoner Tea-Party war der Konflikt mit England von der Streitfrage bestimmt, wie weit die Expansion der landhungrigen Siedler ins Indianerland vorgetrieben werden dürfe. Georg III. hat mit einer Proklamation vom 7. Oktober 1763 die Siedlungsgrenze zuletzt definitiv auf dem Apalachen-Kamm gezogen und die Beachtung dieser Grenze allen Siedlern zur Pflicht gemacht. Diese waren jedoch nicht bereit, die Grenzziehung anzuerkennen, weil sie ihnen ein Hindernis für wirtschaftliches Fortkommen und freies Unternehmertum bedeutete.

Die unbegrenzte und ungesetzliche Landnahme im Westen legte die noch heute gültigen Grundlagen des amerikanischen Credo, der Gesamtheit der zentralen Aussagen zum politischen und wirtschaftlichen Verfassungsbegriff, von denen das amerikanische Freiheits- und Menschenrechtsverständnis die wichtigste ist. Aus dem Credo folgte die Weigerung, den Indianern einen Status des friedlichen Zusammenlebens mit den eingewanderten Siedlern auf der Basis gleicher Rechte auch nur prinzipiell in Aussicht zu stellen. Die amerikanische Landnahme im Westen war, oberflächlich betrachtet, eine Variante der europäischen Expansionen, der mittelalterlichen Ostkolonisation oder der neuzeitlichen Kolonialisierung in Afrika, Lateinamerika und Asien. Tatsächlich aber ging die amerikanische Expansion in Ziel und Reichweite über die europäischen Kolonisationen weit hinaus insofern, als sie keine Missionierung der indianischen Völker bezweckte. Die Landnahme im Westen zielte auf Wachstum von Wirtschaft, Handel und Gesellschaft auf der Grundlage der Nichtintegration der Indianer, die als Störfaktor für diese Ziele betrachtet wurden. Der den Siedlern von der britischen Krone gegebene Auftrag, die neuen Länder unter Schonung und in Respektierung der Rechte der vorgefundenen Völker zu besiedeln und die Indianer für den neuen christlichen Glauben zu gewinnen, wie es noch William Penn 1681 bestimmt hatte, wurde als Wachstums- und Entwicklungshindernis und direkter Eingriff des Staates in die legitimen Rechte der Siedler abgelehnt.

Das amerikanische Dogma von der freien Eigentumsverfügung der Neusiedler über das Land der Indianer, die nicht nur, wie die Afrikaner, als unzivilisiert, sondern als nicht zivilisationsfähig erklärt wurden, ist mehr als nur ein Indiz dafür, wie stark das amerikanische Menschenrechtsverständnis von Anfang an ökonomisch begründet war. Das Dogma, das die Weigerung der Anerkennung der Indianer als Rechtssubjekte zur Voraussetzung hatte, half ein Gruppendenken zu begründen, das der Ausbildung und Anerkennung universaler Rechtsprinzipien nicht förderlich war. Für dieses Denken hat die Plantagenwirtschaft, mit Hilfe aus Afrika eingeführter Sklaven noch unter britischer Regie begonnen, Grundlagen gelegt. Denn der chronische Arbeitsmangel auf den Plantagen der Südstaaten konnte zu keiner Zeit wirklich behoben werden. Die Plantagenwirtschaft zwang die Siedler, Skla- 
verei auf Dauer zu legitimieren, selbst in "God's own country". Nur eine Minderheit der Siedler lehnte die Sklaverei kategorisch als eines Christen nicht würdig ab. Es ist noch heute beeindruckend zu lesen, mit welchen Gründen die Mennoniten in Pensylvanien 1688 gegen die Sklaverei öffentlich Stellung genommen haben.

Das Dogma von der freien Eigentumsverfügung über schwarze Sklaven erleichterte es den Siedlern, die illegale Landnahme im Westen zu legitimieren und sie später durch die Ausruf ung der staatlichen Unabhängigkeit der Vereinigten Staaten zu legalisieren. Der Dissens in einer grundlegenden Frage der Menschenrechte, der Frage der Partizipation der Indianer an diesem neuen Staat, blieb jedoch bis weit in das 20. Jahrhundert hinein bestehen. Er hat die USA nicht gehindert, zur führenden Industrie- und Weltmacht aufzusteigen. Mit dem Aufstieg zur Weltmacht ist der Genocid an den Indianern untrennbar verbunden. Er ist ebensowenig wie die Sklaverei durch die amerikanische Erfolgsgeschichte getilgt worden. Davon zeugte zuletzt die Bürgerrechtsbewegung Martin Luther Kings und zeugen heute die öffentlichen Debatten über das Ob und das Wie einer Entschädigung, die den Nachkommen der indianischen Völker zuteil werden soll für das an ihnen begangene Unrecht. Auch die neuerdings entfachte Kontroverse, ob sich der amerikanische Präsident bei den Nachkommen der Negersklaven für Sklaverei und alle daraus resultierenden Ungerechtigkeiten förmlich entschuldigen soll, ist Teil dieser Debatten um Statusverweigerung und Diskriminierung, die das amerikanische Credo der freien Eigentumsverfügung begründet haben.

Die Tatsache, daß die Eroberung und die uneingeschränkte Verfügungsgewalt des einzelnen über das von ihm eroberte und besiedelte Land den amerikanischen Freiheitsbegriff ausmachen, ist niemals öffentlich eingestanden worden. $\mathrm{Zu}$ diesem Freiheitsbegriff gehört, daß Sklaven als lebendes Inventar auf Grund und Boden verstanden wurden und aus diesem Grunde am Freiheitsbegriff der Siedler nicht teilhaben sollten. Zum amerikanischen Freiheitsbegriff gehört auch, daß Grund und Boden in ihrer Nutzung als nicht durch Rechte der indianischen "Vorbesitzer" beeinträchtigt betrachtet wurden. Beides hat das amerikanische Credo bis heute nicht prinzipiell in Frage gestellt. Das Credo erfährt heute seine Verteidigung im freien Unternehmertum, für das die Kategorien der Freiheit des Welthandels, der uneingeschränkten Investitionsfreiheit und des freienWettbewerbs über alle politischen, territorialen, sozialen, traditionellen und kulturellen Grenzen hinweg die Metaphern sind.

Es ist dieser Begriff der absoluten wirtschaftlichen Unternehmensfreiheit, der die USA in der Begegnung mit fremden Völkern und Kulturen, die europäischen eingeschlossen, in Konflikte verwickelt und sie Mißverständnissen aussetzt. Zwar regiert auch in anderen Ländern der homo oeconomicus; Freiheit ist aber dort in den verschiedenen historischen Epochen und unter den wechselnden politischen und wirtschaftlich-sozialen Bedingungen nicht als maximale Verfügungsgewalt des einzelnen über sein wirtschaftliches Eigentum erfahren worden. Die zunehmende Reduzierung des Freiheitsbegriffs auf eine primär ökonomische Verfügungsgewalt des einzelnen über sein Eigentum, heute verstanden als wei- 
testgehende Verfügungsgewalt über alles, was sich irgendwie zu Geld machen läßt, ist deshalb auch ein Schlüssel zum Verständnis der gegenwärtigen Defizite in der Umweltund Klimapolitik. Sie besagt, daß es für den in Kategorien der Globalisierung denkenden freien Unternehmer eine Grenze seiner Unternehmensfreiheit nicht geben soll. Diese Ideologie liegt wie ein Stein auf zweihundert Jahren amerikanischen Verfassungsdenkens, für das die Herausgeber der Dokumentensammlung bemerkenswerte Beispiele zusammengetragen haben. Sie behindert auch die Anpassung der universalen Menschenrechte an die veränderte Wirklichkeit unter den Herausforderungen eines zeitgemäßen Klimaschutzes. 